\title{
SHORT COMMUNICATION Neuromagnetic evidence for early semantic access in word recognition
}

\author{
Friedemann Pulvermüller, ${ }^{1}$ Ramin Assadollahi $^{2}$ and Thomas Elbert ${ }^{2}$ \\ ${ }^{1}$ MRC Cognition \& Brain Sciences Unit, 15 Chaucer Road, Cambridge, CB2 2EF, UK \\ ${ }^{2}$ Fachgruppe Psychologie, University of Konstanz, 78457 Konstanz, Germany
}

Keywords: evoked magnetic field, lexicon, MEG, semantics, word processing

\begin{abstract}
Magnetic brain responses recorded in the human magnetoencephalogram (MEG) distinguished between words with different semantics but carefully matched for frequency and length. Multiple recordings from a single subject showed that 100 ms following stimulus onset, significantly stronger neuromagnetic responses were elicited by words with strong multimodal semantic associations than by other word material. At this early processing step, there was a highly significant correlation (0.80) between the magnitude of brain responses to individual words recorded over parieto-occipital areas and their semantic association strengths. Subsequent to this early difference related to word meaning, additional differences in MEG responses emerged for words from different grammatical categories. Together, these results suggest that word meaning can be reflected by early neuromagnetic brain responses and before the grammatical information about the word is encoded.
\end{abstract}

\section{Introduction}

One of the most challenging questions in the cognitive neuroscience of language addresses the specific types of information that become available when a word is being comprehended. If a letter string is perceived as a word, does the information about the word's form, grammar and meaning become available at once or in successive steps? Would grammatical information become available before the words' meaning (semantics), or vice versa? We address this question by investigating magnetic brain responses to words belonging to different grammatical and semantic categories and ask whether the earliest physiological distinction would be between grammatical or between semantic word categories.

\section{Methods}

\section{Subjects}

The subject was a 21-year-old right-handed monolingual female student with 15 years of formal education. She had normal eyesight and no history of neurological disease or drug abuse. She was paid for her participation. A single-subject study was preferred to a group study for the following reasons: (i) The latency of evoked brain responses related to cognitive processing varies substantially as a function of stimulus familiarity and, consequently, between experiment participants (Osterhout et al., 1997). (ii) Structural and functional brain organization differ considerably between individuals (Damasio, 1995), thus causing devastating between-subject variance in both topography and timing of evoked fields. Although at present most imaging studies concentrate on group averages, rather than

Correspondence: Friedemann Pulvermüller, as above.

E-mail: friedemann.pulvermuller@mrc-cbu.cam.ac.uk

Received 3 April 2000, revised 26 October 2000, accepted 30 October 2000 thoroughly investigating the reproducibility of results in individual subjects, the single-case strategy appears to us to be as important in cognitive neuroimaging as it undoubtedly is in the study of single patients in cognitive neuropsychology (Caramazza, 1986).

\section{Stimuli}

All words were short and common; their length was 3-7 letters (average, 4.9) or 1-2 syllables (average, 1.5) and their log word frequency (base-ten logarithm of the number of occurrences per million words of standard text) ranged between 1.00 and 2.51 (average, 1.56). There were two types of words, frequently repeated recurrent words and new words. The new words were obtained from recurrent words by replacing one letter. The 16 recurrent words were from four categories: (1) grammatical function words (2) action verbs (3) nouns with strong visual associations and (4) nouns eliciting both visual and action associations. As Table 1 illustrates, categories (1-3) differed regarding their grammatical category. Category (4) was also grammatically distinct from (1) and (2), but differed from (3) only with respect to semantic features. Word frequencies and lengths were matched between the four word categories (average log word frequencies and lengths for each category: (1) 1.57 and 4.8 ; (2) 1.57 and 4.8; (3) 1.55 and 4.8; (4) 1.55 and 5). A behavioural rating study was carried out with 10 native speakers of German. They rated all words on 10-point scales, thereby confirming the word classification into four semantic categories. A significant interaction of the factors word category and association modality (visual vs. action), $F_{3,27}=45.54, \quad P<0.00001$, showed that strong associations of actions were only reported for the stimuli in categories (2) and (4) and strong visual associations were reported for (3) and (4) (Fig. 1). Thus, category (1) showed no associations (amodal), (2) and (3) elicited associations in one modality (unimodal), and (4) in more than 
one (multimodal). Overall semantic association scores were computed as the sum of action and visual ratings.

\section{Procedure}

Within 4 weeks, there were 12 recording sessions, each including three 'runs', each of which included 20 blocks. In each block, 16 recurrent words and two new words were shown in randomized sequences. Prior to the investigation, the participant was familiarized with the recurrent stimuli. She was told to memorise them and to respond by button press (left index finger) only to stimuli not in the memorised set. The task was applied to force the subject to keep the recurrent word set in active memory, and to assure sustained attention to the stimuli. The subject reported that, after reading the short word list a few times, she easily remembered the words without applying mnemonic strategies. Free recall was error-free before each session.

All words were presented tachistoscopically (for $100 \mathrm{~ms}$ ) written in white uppercase letters (maximum word size $7 \times 3 \mathrm{~cm}$ ) on a grey screen $1.4 \mathrm{~m}$ away from the eyes. Stimulus onset asynchronies randomly varied between 1.4 and $2.0 \mathrm{~s}$. A fixation cross appeared in the middle of the screen whenever no word was visible. A Sharp LCD projector outside the magnetoencephalogram (MEG) chamber was used to project the stimuli onto the screen inside.

\section{MEG Recording}

Neuromagnetic signals were recorded $(0.1-100 \mathrm{~Hz}$ bandpass, $508 \mathrm{~Hz}$ sampling rate) using a 148-channel whole-head magnetometer

TABLE 1. Lexical category and basic meaning features of the four word groups

\begin{tabular}{llll}
\hline & \multicolumn{2}{l}{ Meaning } & \\
\cline { 2 - 4 } $\begin{array}{l}\text { Lexical } \\
\text { Category }\end{array}$ & Amodal & Unimodal & Multimodal \\
\hline Function word & $(1)$ & - & - \\
Verb & - & $(2)$ & - \\
Noun & - & $(3)$ & $(4)$ \\
\hline
\end{tabular}

The four word groups used in the experiment differed in their lexical categories and meaning. Lexical distinctions are in rows and meaning-related differences predict major differences in columns.

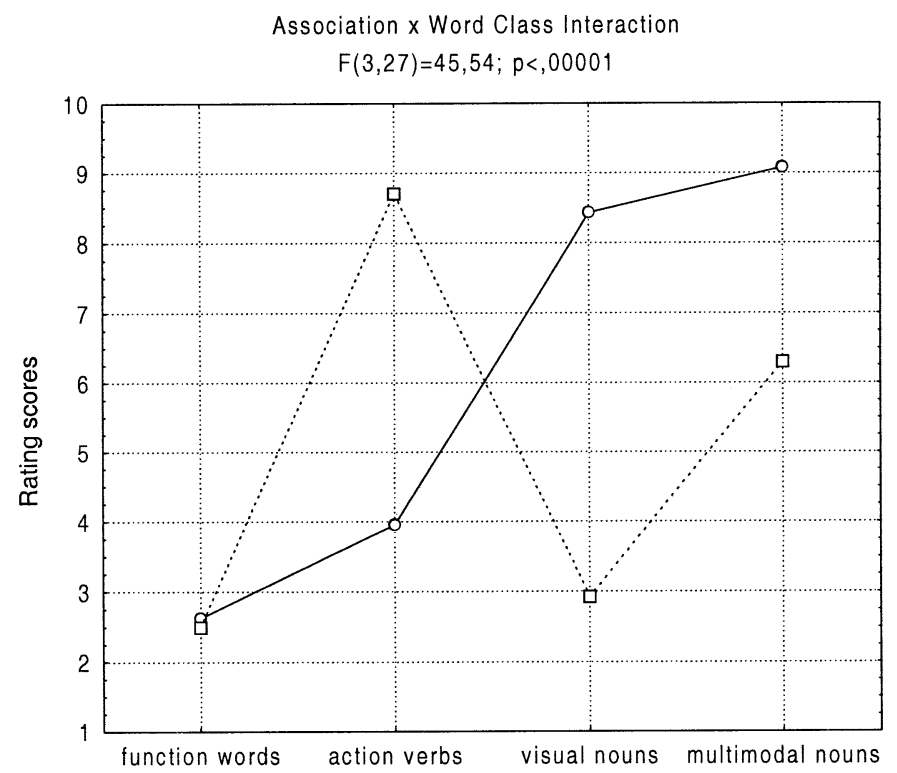

(MAGNES 2500, Neuroimaging, San Diego, CA, USA). Vertical and the horizontal electrooculograms (EOG) were also recorded. The sensor array was always positioned over the same landmarks of the subject's head.

\section{Data analysis}

Raw MEG data were submitted to a global noise filter, split into epochs, and artifacts were rejected (thresholds: EOG $>100 \mu \mathrm{V}$, MEG > $5 \mathrm{pT}$ ). Data from one session were excluded from analysis because of high rejection rates (30\%). For each recording session and word category, stimulus-triggered evoked magnetic fields (evoked fields, EF) were calculated relative to a $100-\mathrm{ms}$ baseline. For statistical analysis, data from channels with high signal-to-noise ratios and hence strong EFs ( $>100 \mathrm{fT}$ ) were selected. The loci from which data were analysed were over left and right parieto-occipital cortex and at the parieto-occipital midline (Fig. 2). Each locus included five adjacent coils from which root mean square values were calculated. Greenhouse-Geisser-corrected ANOVAs $(3$ loci $\times 4$ word categories) were calculated to assess significance of betweencategory differences. For correlation analysis, averaged neuromagnetic signals obtained for each of the 16 words was z-transformed to normalize for any difference related to word length or frequency (Osterhout et al., 1997). The normalized values were correlated with the semantic ratings obtained in the preliminary experiment.

\section{Results}

\section{Behavioural data}

Between 95 and $98 \%$ correct responses were made in the individual sessions. The extremely rare false positives were equally distributed over the recurrent words indicating that all words were remembered equally well.

\section{Physiological data}

Figure 2A shows the distribution of word-evoked fields over the head. Figure $2 \mathrm{~B}$ presents recordings from the left parieto-occipital area for all four word categories. The grand average indicates betweencategory differences, already apparent $\approx 100 \mathrm{~ms}$ after stimulus onset. Statistical analyses were based on an early component, presumably an
- - visual associations …. action associations
FIG. 1. Semantic association ratings for the four word categories on a 10-point scale: only minor semantic associations were reported for function words. Only strong action associations were reported for verbs, and strong visual associations only for the visually related nouns. The multimodal noun category received strong visual association ratings and also substantial scores for action associations. 

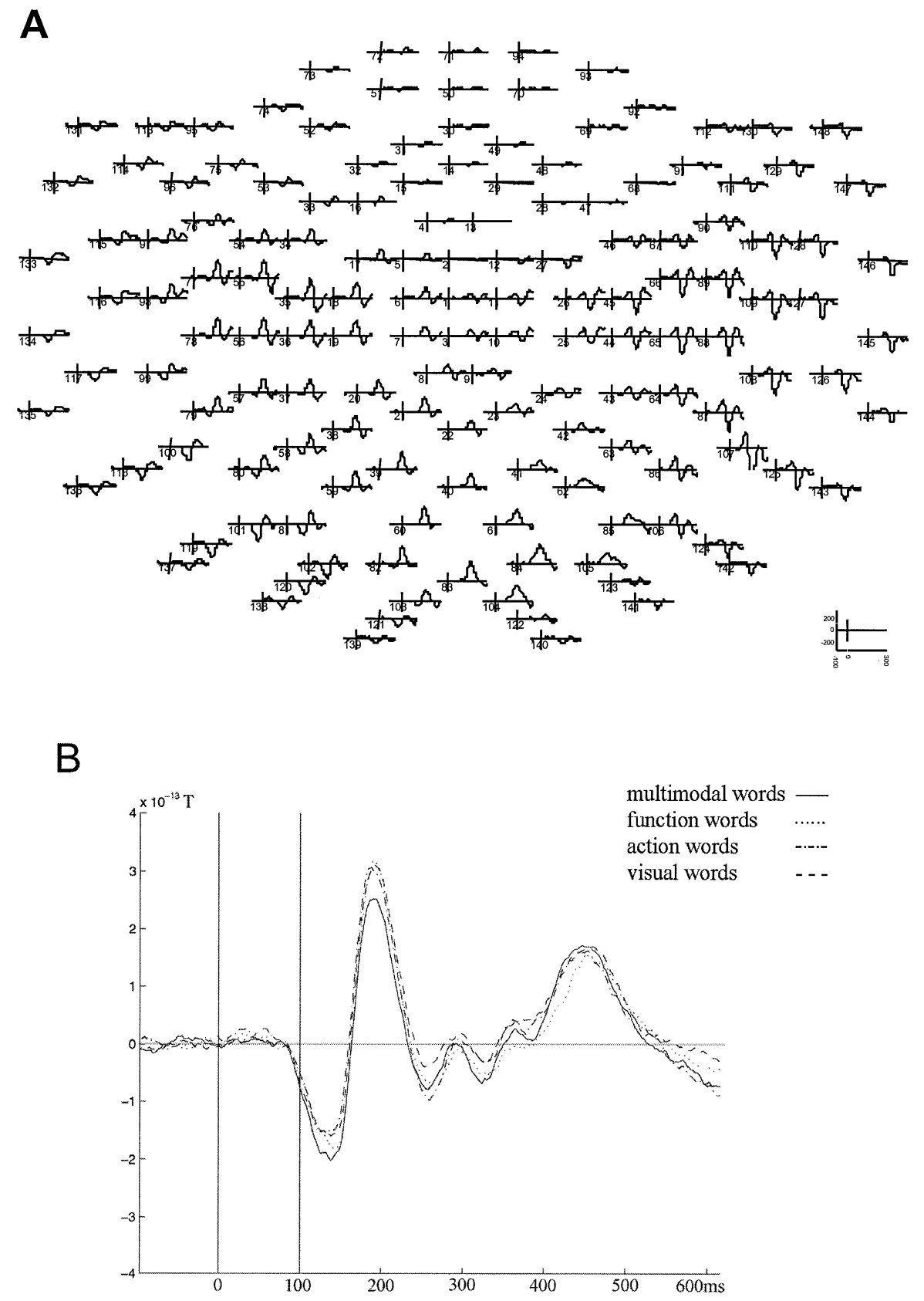

FIG. 2. (A) Neuromagnetic responses evoked by words recorded at 148 MEG channels. The nose is up and left is left. (B) By $\approx 100 \mathrm{~ms}$ after stimulus onset, neuromagnetic responses differed between word categories.

$\mathrm{N} 1 \mathrm{~m}$, the magnetic counterpart of a component of the event-related potential called N1. Average values from the time window $90-120 \mathrm{~ms}$ revealed a significant main effect of word category: $F_{3,30}=5.51$, $\varepsilon=0.93, P=0.005$ (Fig. 3A). Planned comparisons showed significant differences between multimodal nouns [word category (4)] and all other word groups [(4) vs. (1): $F_{1,10}=6.2, P=0.03$; (4) vs. (2): $F_{1,10}=15.3, P=0.002$; (4) vs. $\left.\left.(3): F_{1,10}=6.8, P=0.02\right)\right]$. There was no significant difference between any two of the other three categories. Thus, there was a physiological distinction exactly along the boundary of one semantic category. Words with strong multimodal associations were isolated neuromagnetically from other words, regardless of whether they belonged to the same lexical category or not.
Correlation analyses further confirmed that semantic associations were reflected in neuromagnetic signals. For the early time window around $100 \mathrm{~ms}$, normalized average physiological values obtained for each individual word were plotted against the strength of semantic associations, as revealed by the preliminary experiment. This yielded a highly significant correlation between semantic association strength and amplitude of neuromagnetic responses $(r=0.796, \mathrm{t}=4.93$, $P=0.0002$ ). In the regression plot in Fig. 3C, the four dots representing the amodal function words are on the lower left and those representing multimodal nouns cluster on the upper right. Dots corresponding to unimodal nouns and verbs amalgamate in the middle. This strong correlation leaves little doubt that semantic 
A

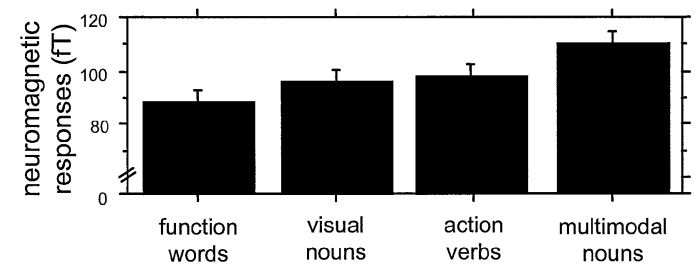

B

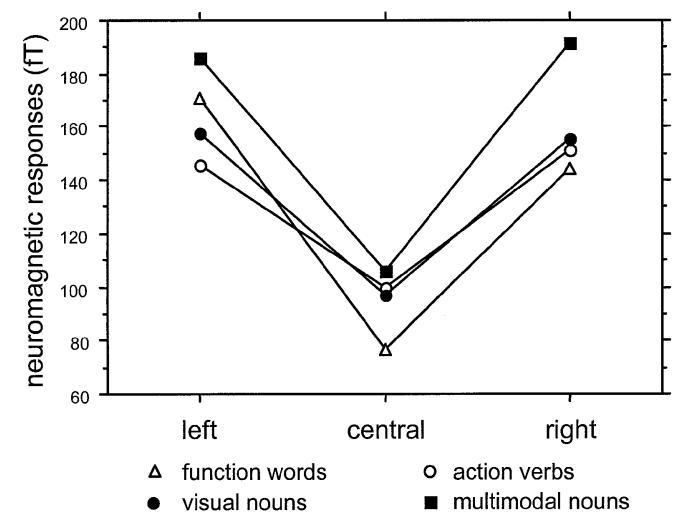

C

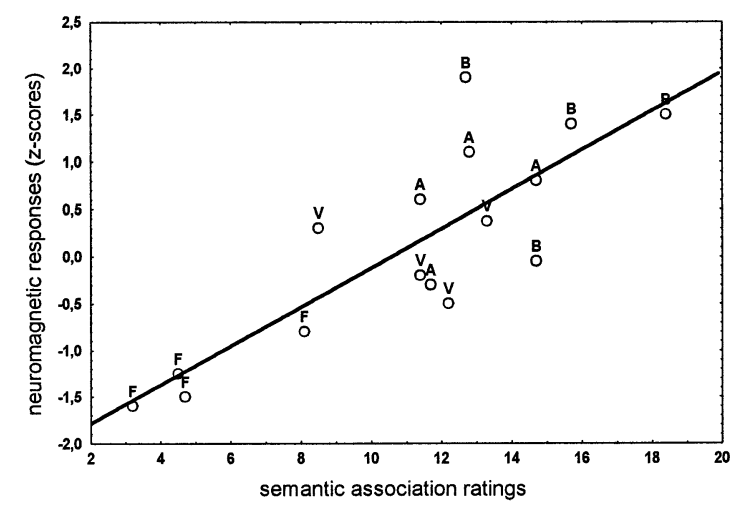

FIG. 3. Statistical analyses of neuromagnetic responses revealed early meaning-related differences between words. (A) Significant main effect of word category in the 90-120 ms time window. (B) Significant interaction of word category and locus (left hemisphere, midline, right hemisphere) in the 120-160 ms time window.(C) Significant correlation $(r=0.796)$ of semantic association strength and magnitude of neuromagnetic responses in the 90 $120 \mathrm{~ms}$ window. Each dot represents one word (F, function word; A, action verb; V, visually-related noun; B, bi- or nultimodal noun).

aspects of the words became manifest in the neuromagnetic activity early $(\approx 100 \mathrm{~ms})$ after stimulus onset.

In a later time interval, $120-160 \mathrm{~ms}$ after stimulus onset, there was a significant interaction of the factors word category and locus, $F_{6,60}=4.04, \varepsilon=0.431, P=0.02$ (Fig. 3B). At left-hemispheric recordings, all word categories diverged from the multimodal category which again produced the strongest signals. Over the midline, function words produced significantly weaker responses compared to all other groups, whilst over the right hemisphere only the multimodal words separated from the rest $(21.6>F>4.7,0.0009<P<0.05)$. At this later time interval, word category differences in neuromagnetic responses thus varied with recording site. Consistent with earlier findings (Pulvermüller et al., 1995), there was a significant left-right difference for function words (stronger signals over the left hemisphere than over the right; $F_{1,10}=5.80, P=0.03$ ), but not for any of the other word groups.

\section{Discussion}

Neuromagnetic responses from a single subject already reflected word semantics $100 \mathrm{~ms}$ after onset of visually presented word stimuli. Nouns with multimodal associations evoked stronger magnetic fields than did other words, regardless of whether they belonged to the same or different lexical categories. At this early point in time, semantic association strength was correlated with the magnitude of neuromagnetic responses. Some $50 \mathrm{~ms}$ later, $\approx 150 \mathrm{~ms}$ after stimulus onset, magnetic brain responses revealed additional physiological distinctions of the categories of nouns, verbs and function words over the left hemisphere. These results show that semantic information about a word under processing can become apparent in neuromagnetic responses before there is evidence for grammatical distinctions.

These results replicate aspects of earlier findings about wordcategory differences revealed by large-scale imaging techniques. Using naming tasks, Damasio et al. (1996) and Martin et al. (1996) found differences in local cerebral blood flow between tool and animal names. The present MEG data from a memory retrieval task confirmed such differences between nouns with unimodal visual associations (including most animal names) and nouns with associations in more than one modality (visual and motor, including most tools). Electrocortical differences between lexical categories, such as function words, verbs and nouns, had been reported in earlier EEG studies (e.g. Neville et al., 1992; Dehaene, 1995; Pulvermüller et al., 1995). These studies detected word category differences at $\geqslant 150 \mathrm{~ms}$ after stimulus onset.

The present study has, we believe, a potentially important methodological implication. Our results indicate that the earliest neurophysiological indicators of cognitive brain processes can best be investigated in single subject studies where one subject is examined numerous times. Detailed physiological investigation of healthy individuals can be used to draw careful conclusions on the organization of the brain-internal lexicon, in the very same way that observation of single neuropsychological patients who show, for example, category-specific difficulties consistent with grammatical or semantic distinctions can illuminate the neurobiological basis of cognition.

However, the single-subject design may not have been the only reason why early cognition-related brain activity was revealed by this study. In the present recognition task, the relevant memory traces for words were, most probably, already active when the word stimuli were presented (active memory; Fuster, 1997), and less time may therefore have been necessary to fully ignite them. Further, Skrandies (1998) reported early physiological differences between semantic word categories obtained from a group of subjects who looked at and memorised new words. Therefore, the single case design appears helpful but not indispensable for obtaining early $(\approx 100 \mathrm{~ms})$ physiological correlates of word semantics.

One may claim that not their visual and action associations but an additional unknown property of the word groups may have been responsible for the early divergence of brain responses. However, we re-emphasize that the words were carefully matched for important psycholinguistic variables (including word length and frequency), which rules them out as possible confounds. In addition, the words' concreteness or imageability cannot account for the difference, because all nouns selected were rated as equally concrete and imageable. Therefore, concreteness/imagery alone would not allow distinction between the visually related and the multimodal noun categories. The only variable accounting for the early difference in neuromagnetic responses appears to be the cumulative semantic association strength obtained for different 
modalities. Further, there was no evidence that different mnemonic strategies were applied for different words, arguing against the possibility that putative mnemonic differences contributed to differential brain responses to words.

The relative timing of syntax and semantic processing has been investigated in numerous studies. Event-related potentials indicated that, in sentence processing, syntactic anomalies can be reflected in a late positive component (P600; Osterhout \& Holcomb, 1992), whilst semantic violations usually elicited an earlier response starting $\approx 300 \mathrm{~ms}$ (N400; Kutas \& Hillyard, 1983). The earliest grammatically related brain response was a wave elicited by syntactic anomalies (phrase structure violations) which peaked at $125 \mathrm{~ms}$ (Neville et al., 1991). The present results confirm that syntactic distinctions can be reflected in neurophysiological responses around 120-150 ms after stimulus onset. However, the data also show that the earliest linguistically related brain responses recorded so far, at $\approx 100 \mathrm{~ms}$ after visual stimulus onset, reflected aspects of word semantics.

The early category-specific neuromagnetic difference between multimodal and other words, the early correlation of brain responses with word semantics, and the later physiological distinction of nouns and verbs, cannot be explained by models postulating that the processing of the meaning of a word is a late process. Some psycholinguistic models (e.g. Caplan, 1996) postulate that semantic access is actually the last process in word comprehension, following access to the word form in the lexicon, where some of its grammatical properties are stored. The present results provide an existence proof that semantic information can become physiologically apparent before grammatical information characterizing words. They are consistent with models proposing that the processing of syntactic aspects of words follows semantic access (Pulvermüller, 1999, 2000) and add to earlier observations that the neurophysiological signs of aspects of semantics can precede syntactic brain processes (Brown et al., 2000).

Finally, we wish to highlight implications of this study for brain models of cognitive processing. Stimulus-evoked neuronal activity in the primary visual area can be detected $\approx 50 \mathrm{~ms}$ after visual stimulation (cati et al., 1988). Only another $\approx 50 \mathrm{~ms}$ later we found a difference between physically similar words with different meanings. Being of equal length and similar shape and luminance, these stimuli activate the same sensory pathway to a similar degree. To account for the differential cortical activation they elicited, it is necessary to assume that cortico-cortical circuits were effective. It may be that the stronger semantic associations of multimodal words are laid down in the cortex as strong links between numerous widely scattered neurons. This may provide a tentative explanation for the present data. Fast feedforward and feedback activation within distributed cell assemblies representing words (Pulvermüller, 1999) may be the cause of the early divergence of word-evoked neuromagnetic fields.

\section{Acknowledgements}

We thank Patrick Berg and Christian Wienbruch for their help and three anonymous referees for their comments on earlier versions of this manuscript. Supported by grants $\mathrm{Pu} 97 / 5-2$ and $\mathrm{Pu} 97 / 11-1$ of the Deutsche Forschungsgemeinschaft (D.F.G.).

\section{Abbreviations}

EF, evoked fields; EOG, electrooculogram; MEG, magnetoencephalography, magnetoencephalogram.

\section{References}

Brown, C., van Berkum, J.J.A. \& Hagoort, P. (2000) Discourse before gender: an event-related brain potential study on the interplay of semantic and syntactic information during spoken language understanding. J. Psycholing. Res., 29, 53-68.

Caplan, D. (1996). Language: Structure, Processing, and Disorders. MIT Press, Cambridge, MA.

Caramazza, A. (1986) On drawing inferences about the structure of normal cognitive systems from the analysis of patterns of impaired performance: the case for single-patient studies. Brain Cognit., 5, 41-66.

Damasio, H. (1995). Human Brain Anatomy in Computerized Images. Oxford University Press, New York, Oxford.

Damasio, H., Grabowski, T.J., Tranel, D., Hichwa, R.D. \& Damasio, A.R. (1996) A neural basis for lexical retrieval. Nature, 380, 499-505.

Dehaene, S. (1995) Electrophysiological evidence for category-specific word processing in the normal human brain. Neuroreport, 6, 2153-2157.

Ducati, A., Fava, E. \& Motti, E.D.F. (1988) Neuronal generators of the visual evoked potentials: intercerebral recording in awake humans. Electroencephalogr. Clin. Neurophysiol., 71, 89-99.

Fuster, J.M. (1997) Network memory. Trends Neurosci., 20, 451-459.

Kutas, M. \& Hillyard, S.A. (1983) Event-related brain potentials to grammatical errors and semantic anomalies. Memory Cognition, 11, 539550.

Martin, A., Wiggs, C.L., Ungerleider, L.G. \& Haxby, J.V. (1996) Neural correlates of category-specific knowledge. Nature, 379, 649-652.

Neville, H.J., Mills, D.L. \& Lawson, D.S. (1992) Fractionating language: different neural subsystems with different sensitive periods. Cereb. Cortex, 2, 244-258.

Neville, H., Nicol, J.L., Barss, A., Forster, K.I. \& Garrett, M.F. (1991) Syntactically based sentence processing classes: evidence from eventrelated brain potentials. J. Cognit. Neurosci., 3, 151-165.

Osterhout, L., Bersick, M. \& McKinnon, R. (1997) Brain potentials elicited by words: word length and frequency predict the latency of an early negativity. Biol. Psychol., 46, 143-168.

Osterhout, L. \& Holcomb, P.J. (1992) Event-related brain potentials elicited by syntactic anomaly. J. Mem. Lang., 31, 785-806.

Pulvermüller, F. (1999) Words in the brain's language. Behav. Brain Sci., 22, 253-336.

Pulvermüller, F. (2000) Syntactic circuits: How does the brain create serial order in sentences? Brain Lang., 71, 194-199.

Pulvermüller, F., Lutzenberger, W. \& Birbaumer, N. (1995) Electrocortical distinction of vocabulary types. Electroencephalogr. Clin. Neurophysiol., 94, 357-370.

Skrandies, W. (1998) Evoked potential correlates of semantic meaning - a brain mapping study. Cogn. Brain Res., 6, 173-183. 